\title{
Abstracts from the Biofilm Club meeting held at the School of Pharmacy, University of Manchester on 4 November 2004
}

* Corresponding author: G. H. Markx

E gerard.markx@manchester.ac.uk

1 School of Chemical Engineering and Analytical Science, University of Manchester, Sackville Street, Manchester M60 1OD, UK

2 NERC Centre for Ecology and Hydrology, Environmental Biotechnology, Mansfield Road, Oxford OX1 3SR, UK

3 Department of Civil and Structural Engineering, University of Sheffield, Mappin Street, Sheffield S1 3JD, UK
* Corresponding author: A. J. McBain

E andrew.mcbain@manchester.ac.uk

School of Pharmacy and Pharmaceutical Sciences, University of Manchester, Oxford Road, Manchester M13 9PL, UK

\section{Microfabrication of biofilms}

\author{
G. H. Markx ${ }^{1 *}$, B. Alp ${ }^{1}$, J. S. Andrews ${ }^{1,3}$, \\ C. Gonzalez-Ramirez ${ }^{1}$, V. P. Mason ${ }^{1,2}$, \\ G. M. Stephens ${ }^{1}$, I. P. Thompson ${ }^{2}$ and K. Zhu ${ }^{1}$
}

Biofilms have many of the properties of eukaryotic tissues. Microfabrication approaches have been used in tissue engineering as a "bottom-up" approach for the rapid construction of tissue equivalents. Similar approaches can be used for constructing biofilms with defined internal architectures. We have shown that electric fields can be used to create three-dimensional biofilms with known spatial organization.

First, using photolithography, microelectrodes are fabricated onto the surface of microscope slides, which produce a very high electric field strength in specific regions between the electrodes when energized with relatively small AC voltages (typically 5-20 V peak to peak, $1 \mathrm{MHz}$ ). A chamber is created on top of the microscope slide from a cover slip. When cells, suspended in deionized water or low conductivity buffer solutions, are introduced into this chamber, they are drawn to the high electric field regions by a phenomenon called dielectrophoresis. Different species or strains of microorganisms can be put next to each other or on top of each other, by addressing individual electrodes selectively, or by sequentially introducing the different microorganisms. A further immobilization step, using gels or cross-linking agents, ensures that cells stay in place once the electric field has been switched off. The cells in the biofilms have been shown to be viable, active and able to grow. By using microorganisms in which the production of green fluorescent protein is linked to external factors it is possible to study the effect of the microenvironment on the cells.

So far, we have used such approaches to study the exchange of metabolites, signalling molecules (acetyl homoserine lactones) and plasmids between cells within biofilms. Also, we have investigated the effects of spatial organization on the penetration of biocides into the biofilm.

\section{Modelling drain biofilms}

\section{A. J. McBain* and P. Gilbert}

Clinical epidemiologists have long recognized the potential of sink drains in hospital wards to harbor and disseminate pathogens, yet the microbial composition of this environment remains a "black hole". Complex biofilm communities can be found in the sink drains of hospitals, domestic and catering kitchens, bathrooms and food factories. In a survey of domestic sink drain outlets in the Manchester area, for example, drain biofilms generally harbored between 10 and $11 \log _{10}$ viable cells/g, mainly enteric species and pseudomonads. Sink drains are the final recipients of many antibacterial 
GlaxoSmithKline, St George's Avenue, Weybridge, Surrey KT13 ODE, UK

E andrew.x.middleton@gsk.com hygienic consumer products. Concern that the increased domestic use of biocides such as triclosan (TCS) in consumer products might contribute to the emergence of antibiotic resistance, and a bias in the literature towards pure culture studies, led us to devise an in vitro model of the domestic drain for the study of biocide exposure in realistic microbial communities.

Sink-drain microcosms were established by using excised biofilms from two separate drains to inoculate constant-depth film fermenters (CDFFs) that were fed intermittently with artificial dishwater and constantly exposed to untreated tap water. Differential plate counts, live-dead direct counting and denaturing gradient gel electrophoresis showed that the major bacterial species from in situ material were stably maintained in the microcosms and that bacterial population densities resembled those found in situ. Exposure of the drain microcosms to TCS or quaternary-ammonium-containing domestic detergents at normal use levels had little effect upon total bacterial viability but did not cause antibiotic (10 test agents) or biocide resistance to emerge, even over prolonged exposure (up to 6 months). Rather, organisms with innate insusceptibility or tolerance, including aeromonads, pseudomonads, stenotrophomonads and Alcaligenes species became clonally expanded during extended biocide exposure. These studies suggest that low-level exposure of complex environmental biofilm communities to biocides does not necessarily affect antimicrobial susceptibility, possibly owing to a combination of natural tolerance, ecological competition, degradation and penetration failure.

The CDFF has proved to be a valuable tool for maintaining realistic, colonization-resistant drain biofilms and has enabled this ecosystem to be continuously modeled in our laboratory for over 5 years.

\section{Interaction between mycobacteria and the respiratory mucosa}

\section{A. M. Middleton}

The interaction of respiratory pathogens with mucosal surfaces of the respiratory tract is thought to be critical in their pathogenesis. Organ cultures can be used to study the initial interaction of bacteria with the mucosal surface and subsequent events, in particular the effects of bacteria on the structure and function of the respiratory epithelium.

Organ cultures have a near-normal ratio of differentiated cell types that maintain their three-dimensional relationships with the extracellular matrix and submucosal tissues. By incorporating an air interface they closely simulate in vitro the physiological conditions of the respiratory mucosa found in vivo.

The organ culture model can remain structurally and functionally intact for up to 3 weeks, with maintenance medium replaced daily. Tissue can be removed from the model and examined by light microscopy, scanning electron microscopy and transmission electron microscopy. Organ cultures are sufficiently sensitive to discriminate between the interactions of closely related bacterial strains and so provide a powerful tool by which to investigate the importance of a bacterial toxin or a surface structure involved in adherence during infection of the mucosa.

The model has been used with an air interface to investigate the interaction of Mycobacterium species with human bronchial tissue. Despite differences in their clinical virulence, Mycobacterium avium complex and Mycobacterium tuberculosis demonstrated identical initial adherence to the epithelium. The bacteria adhered to mucus with a fibrous appearance and to areas of epithelial damage. Immunogold labelling was used to investigate the specific adherence in areas of damage, where fibronectin was found to be the target.

Increasing the incubation time of the organ cultures showed that M. avium complex numbers increased on the epithelial surface. However, M. tuberculosis numbers decreased on the surface, but were recovered from within the mucosa. 
Microbiology and Gut Biology, Ninewells Hospital and Medical School, University of Dundee, Dundee DD1 9SY, UK

Eg.omay@dundee.ac.uk
Inhibiting adherence to fibronectin influenced the subsequent infection of respiratory tissue by M. avium complex, but not by M. tuberculosis. This demonstrates that the interaction of $M$. avium complex with fibronectin is a critical factor in the colonization and invasion of the mucosa, whereas M. tuberculosis infection of the mucosa proceeds by different mechanisms, since M. tuberculosis infection was found to be independent of fibronectin adherence.

\section{Acknowledgement}

This work was carried out by the author when he was affiliated to the Host Defense Unit, Royal Brompton Hospital and Imperial College Institute of Science, Technology and Medicine, London, UK.

\section{Modelling the gastrointestinal tract}

\section{G. A. O'May}

The human gastrointestinal tract, and particularly the large bowel, is a prime site for microbial biofilm formation because of its large surface area, high population density and nutrient availability. Such biofilms can influence the health and disease of the host, owing to their proximity to the epithelium. Sampling these communities is problematic because of (a) ethical concerns, (b) the discomfort caused by colonoscopy procedures and (c) the fiscal and temporal cost of clinical studies.

Modelling, therefore, is necessary in order to carry out basic science and to evaluate potential therapeutics. Multi-stage chemostat models allow simulation of the prevailing environmental conditions ( $\mathrm{pH}$, nutrient availability) in the proximal, transverse and distal colon. In this study, a two-stage chemostat within which was suspended mucin-filled traps was inoculated with a $20 \%$ (w/v) faecal slurry from a healthy donor and maintained at $37^{\circ} \mathrm{C}, \mathrm{pH} 5.5$ (vessel 1) and 6.8 (vessel 2). Microbial biofilm formation in mucin gels was assessed by culturing techniques and mucin-degrading enzyme activities in the biofilm and planktonic populations were measured by colorimetric assay. Mucin gels were colonized within $2 \mathrm{~h}$. Bacteroides species were detected in biofilms in both vessels, with the exception of B. caccae, which was present only in vessel 2 and reached levels of approximately $7 \log _{10}$ colony-forming units (c.f.u.)/ml. Enterobacteria and clostridia also colonized mucin gels in both vessels within the initial $6 \mathrm{~h}$ period, reaching levels of approximately $7 \log _{10}$ c.f.u./ml. Bifidobacteria were detected only in biofilms in vessel 2, where they reached approximately $7 \log _{10}$ c.f.u./ml. Profiles of mucin-degrading enzyme activities were markedly different in planktonic and biofilm communities, indicating that these populations were metabolically distinct. Scanning electron micrographs of mucin gels showed that discrete microcolonies were present after $3 \mathrm{~h}$ and total surface coverage was evident after $24 \mathrm{~h}$.

This model system provides a reasonable simulation of mucosal bacterial communities in the human large bowel and is useful for the study of bacterial interactions and their effects on the host.

\section{Modelling oral biofilms}

\footnotetext{
* Corresponding author: J. Pratten

E j.pratten@eastman.ucl.ac.uk

Division of Microbial Diseases, Eastman Dental Institute, University College London, 256 Gray's Inn Road, London WC1X 8LD, UK
}

\section{J. Pratten* and D. A. Spratt}

Dental diseases are site specific and the heterogeneous nature of the plaque biofilm has led to problems with access and sampling. Additionally, there 
* Corresponding author: A. P. Roberts

E a.roberts@eastman.ucl.ac.uk

Division of Microbial Diseases, Eastman Dental Institute, University College London, 256 Gray's Inn Road, London WC1X 8LD, UK are issues associated with ethics for animal and human models. This has led inevitably to the development of laboratory-based models that simulate oral biofilms in vitro. There are many experimental systems available for the generation and study of in vitro dental plaques, such that a suitable system can be chosen to suit the requirements of almost any investigation. All are compromises between the reality of the in vivo ecosystem and the simplification and controllability necessary to gain meaningful, useful results.

Historically, artificial mouth models have been used since the end of the nineteenth century to investigate dental caries in vitro. Since this time, and as researchers realize that they need to study bacteria in the biofilm mode of growth, other models have been developed that have been used additionally to study dental plaque.

For example, the flow cell is often the system of choice for short-term adhesion and colonization studies under shear; it also enables the nondestructive observation of biofilm development in real time. The system can be inoculated either by adding an inoculum to the medium reservoir or by initially passing a culture through the flow cell to allow cells to adhere before feeding with sterile medium takes place. Shear forces are also important in the Modified Robbins device, which additionally allows investigation of a number of substrata (and replicates) over a period of time. In chemostat models there is also a controlled fluid environment and planktonic phase, with a nutritionally limited growth rate that interacts biologically with the biofilm. A large number of replicate samples and a variety of surfaces can be placed within the chemostat and properties such as $\mathrm{pH}$ can be controlled.

Artificial mouths are specifically constructed to mimic the situation in the oral cavity. Hence, dental plaque formation is studied on a human tooth inoculated with saliva and then supplied with mucin-containing artificial saliva. In order to study biofilm development or perturbation, for example by the use of antimicrobial agents over a period of time, data must be comparable and reproducible from experiment to experiment. One approach to reproducibility is to develop constant-depth reactors where surface growth is periodically removed to maintain a constant geometry. Such a device, the constant-depth film fermenter employs a mechanical scraper bar and has been developed to investigate the growth of dental plaque organisms.

Biofilms present in the oral cavity are complex, diverse structures. The organisms contained within them cause a range of diseases, the ecology and pathology of which remain, largely, unknown. The use of laboratory models may help us to understand these varied biofilms in order to combat the diseases they cause.

\section{Modelling antibiotic resistance transfer in oral biofilms}

\section{A. P. Roberts*, G. Cheah, J. Pratten, D. Ready, $M$. Wilson and P. Mullany}

Our recent research efforts have focused on conjugative transposons (CTn) from oral bacteria and their ability to transfer between bacteria of different species. Whilst this can be demonstrated by filter-mating experiments, these do not represent the conditions found within the oral cavity. To simulate this environment we have carried out a series of experiments on oral biofilms grown in the constant-depth film fermenter (CDFF) inoculated with saliva collected from 10 healthy volunteers who had not taken antibiotics in the previous 3 months. 
The initial experiments demonstrated that a Bacillus subtilis containing a Tn916-like, tetracycline-resistance-encoding CTn; Tn5397 could transfer the tetracycline resistance to a member of an established oral biofilm community within $6 \mathrm{~h}$ of the initial inoculation of the B. subtilis donor. Bacillus subtilis could not be isolated from the biofilms after $24 \mathrm{~h}$. However, Tn5397 was still located in a streptococcal host, demonstrating that transient bacteria that are not normally considered part of the normal oral flora may have the ability to transfer genetic information to normal oral bacteria when they pass through the oral cavity on particles of food, etc.

Further experiments have demonstrated that the majority of tetracycline resistance found in bacteria derived from adult saliva is conferred by tet $(\mathrm{M})$ present on Tn916-like CTns. Many of these native CTns have also been shown to be able to transfer to Enterococcus faecalis during filter-mating experiments. Additionally transfer has been observed within the CDFF. In these experiments, a defined tetracycline-sensitive microbial consortia was used, as the background resistance in saliva-derived biofilms made the interpretation of results problematic. This 10 -member microbial consortia was used to inoculate the CDFF together with a tetracycline-resistant $S$. salivarius containing a Tn916-like CTn that had previously been shown to transfer during filtermating experiments. After $150 \mathrm{~h}$ of growth two distinct tetracycline-resistant streptococcal species were isolated, demonstrating that transfer of CTns can occur among the normal flora within the oral cavity as well as between the oral flora and transient bacteria.

* Corresponding author: P. S. Watson E denpsw@leeds.ac.uk

Division of Oral Biology, Leeds Dental Institute, Clarendon Way, Leeds LS2 9LU, UK

\section{Plaque biofilms grown in vivo on natural enamel}

\section{P. S. Watson*, C. Robinson, P. D. Marsh, D. A. Devine, J. Kirkham, R. C. Shore and B. R. Nattress}

Standard in vitro methods used to determine antimicrobial activity are poorly predictive of efficacy against complex multi-species environmental biofilms, and in this context models that produce natural undisturbed biofilms are important. In the field of oral care, several models allow recovery of intact plaque biofilms for study ex vivo. This is valuable in studies of anti-plaque agents used in caries prevention/treatment, as the effectiveness of these agents relies on their uptake and distribution in plaque biofilms. In the case of agents that protect enamel, penetration to the underlying surface is also important. Test systems that use the natural tooth surface as a growth substratum crucially allow the study of interactions between topically applied agents, biofilms and mineralized tissue.

The Leeds In Situ Device comprises a disc of sterilized human enamel, to the natural surface of which is attached a nylon ring that provides a stagnation site for plaque accumulation. Using composite resin, plaque-generating devices may be bonded to the buccal surfaces of molar teeth in the mouths of healthy volunteers. After continuous plaque-accumulation for 4-28 days (during which time the dietary and oral hygiene habits of the device-wearing volunteer may be controlled as required) devices may be de-bonded and recovered intact with undisturbed plaque in situ. Biofilms recovered in this way retain their natural heterogeneous architecture and are similar to interproximal plaque in terms of thickness and microbial composition.

Such biofilms (and the underlying substratum) are amenable to study using a variety of chemical, physical and biological methods. Properties of whole biofilms, such as architecture, cell viability, microbial composition and acidogenicity may be readily studied, for example by confocal microscopy. 
Furthermore, biofilm-processing techniques have been developed that allow plaque biofilms to be sampled and analysed throughout plaque depth. This approach allows the study of crucial mass-transfer phenomena (e.g. the penetration through biofilms of growth substrates, metabolites and antimicrobial agents) and the determination of microbial species distribution.

The use of this in situ model allows the development (and subsequent study) of complex plaque biofilms under naturally varying intra-oral conditions that cannot be replicated using even the most sophisticated in vitro approaches. 